\title{
Metanálise dos efeitos agudos do alongamento na realização de corridas curtas de alta intensidade
}

CDD. 20.ed. 796.4

796.426
Yuri Salenave RIBEIRO* Fabrício Boscolo Del VECCHIO*
*Escola Superior de Educação Física Universidade Federal de Pelotas.

\section{Resumo}

Informações das pesquisas explorando efeitos do alongamento no desempenho em corridas curtas de alta intensidade (CCAI) são controversas. Com isso, esta metanálise objetivou examinar os desfechos decorrentes da execução de diferentes protocolos de alongamentos, prévios à execução de CCAI. A pesquisa foi realizada em diversas bases de dados, usando combinações dos seguintes termos de referência: "sprint" e "stretching". Selecionaram-se estudos com pessoas do sexo masculino e idade superior a 16 anos, sem restrição de modalidade, nível de aptidão física e procedimentos de avaliação utilizados. Após diferentes depurações, localizaram-se 11 investigações como apropriadas para análises, das quais resultaram 62 situações para serem estudadas. Como variáveis dependentes, consideraram-se o Tamanho de Efeito (TE) e o Delta Percentual $(\Delta \%)$, e, como fatores, delineamento adotado, tipo de alongamento, protocolo de avaliação, número de séries, modalidade esportiva, nivel de aptidão e prática pregressa de alongamento. Os resultados sugerem que: a) alongamento dinâmico $(A D)$ promove rendimento significativamente superior quando comparado ao alongamento estático (AE) $(p<0,001)$ ou misto $(A M)(p<0,002)$; b) há diferença no TE e no $\Delta \%$ entre corridas com mudança de direção e corridas lineares (até $20 \mathrm{~m}, \mathrm{p}=0,003$, e acima de $20 \mathrm{~m}, \mathrm{p}<0,009)$; c) realização de vários testes proporciona melhores resultados que aplicação de teste único após aquecer e alongar ( $p=0,001)$; e d) executar série única de alongamento é menos prejudicial que duas $(p=0,016)$ e três séries $(p<0,001)$. Sendo assim, é possivel a obtenção de pequena vantagem incorporando o $A D$ em relação ao $A E_{1} A M$ ou ausência de estímulos para a execução de CCAl.

UnIteRmos: Alongamento; Corridas curtas de alta intensidade; Efeito agudo; Desempenho físico.

\section{Introdução}

Dentre as capacidades físicas que podem ser determinantes para o sucesso no desempenho esportivo se destaca a aptidão neuromuscular, em suas manifestações de força máxima e potência (DE Villarreal, Kellis, Kraemer \& IzQuierdo, 2009; Kraemer, Mazzetti, Nindl, Gotshalk, Volek \& Bush, Marx, Dohi, Gómez, Miles, Fleck, Newton \& HÄKKINEN, 2001). De modo complementar, as práticas que envolvem corridas curtas de alta intensidade (CCAI)/"sprints" devem ser incluídas nesse contexto, dada sua presença nas ações determinantes do êxito competitivo (ZATSIORSKY \& KRAEMER, 2008).

Em geral, no processo de condicionamento para atividades vigorosas, a prática preparatória de exercícios de aquecimento (BISHOP, 2003) e elementos de alongamento (Magnusson \& Renstrom, 2006;
Shellock \& Prentice, 1985) são bastante comuns. Entretanto, a literatura apresenta dados sugerindo que este último, realizado como componente do aquecimento, pode diminuir o desempenho subsequente para movimentos que exigem força máxima e potência muscular, como em "sprints" simples, ou diminuindo a capacidade de sua execução múltipla, em inglês conhecida como "Repeated Sprint Ability" (RSA) (Beckett, Schneiker, Wallman, Dawson \& Guelfi, 2009; Favero, Midgley \& Bentley, 2009; Sayers, Farley, Fuller, JubenVille \& Caputo, 2008; Stewart, Adams, Alonso, Van Koesveld \& CampBell, 2007). A principal característica desses estudos é identificar e quantificar o efeito agudo que os exercícios prévios de alongamento podem provocar na posterior execução da tarefa de CCAI (Fletcher \& Jones, 2004; Sim, 
Dawson, Guelfi, Wallman \& Young, 2009). Tais técnicas de alongamento se baseiam na aplicação de tensão sobre o músculo; ou seja, seus componentes estruturais, inclusive o tecido conjuntivo, têm seus comprimentos aumentados (STONE, Ramsey, Kinser, O'BRYANT, AYERS \& SANDS, 2006).

As informaçōes técnico-específicas são controversas quanto aos desfechos das práticas de alongamento, as quais podem proporcionar efeitos agudos prejudiciais ou benéficos em avaliações motoras subsequentes. De acordo com as informações apresentadas nos trabalhos, registra-se a necessidade de identificação dos elementos que caracterizam as modificaçōes no desempenho, o que torna os tipos de alongamento (estático ou dinâmico) componente viável para interpretação dos achados, em virtude da possibilidade de agrupamento de estudos nos quais foram desempenhados protocolos com os dois tipos diferentes de estímulos: estático ou dinâmico. Esse agrupamento contribui para a análise dos resultados, considerando que diferentes métodos tendem a apresentar efeitos agudos particulares, o que pode ser refletido no resultado das avaliaçōes (HeRMAN \& SMith, 2008). Para o alongamento estático (AE), algumas investigações apontam decréscimo no rendimento em CCAI (AMIRI-KHORASANI, Sahebozamani, Tabrizi \& Yusof, 2010; Fletcher \& ANNess, 2007; Fletcher \& Jones, 2004) e são cogitadas duas razóes: uma relacionada com a rigidez musculotendínea, a qual seria afetada negativamente dificultando e/ou diminuindo o armazenamento de energia elástica para o ciclo de alongamentoencurtamento (WINCHESTER, NELSON, LANDIN Young \& SCHEXNAYDER, 2008), e outra de caráter neural, com a inibição e/ou dificuldade na transmissão de estimulação advinda do sistema nervoso central, dado que o trajeto e atividade dos proprioceptores localizados no músculo seriam prejudicados pelo alongamento (Kistler, Walsh, Horn \& COX, 2010).
Por outro lado, são encontrados resultados demonstrando que técnicas as quais envolvam alongamentos dinâmicos $(\mathrm{AD})$ podem produzir melhor rendimento (Chaouachi, Castagna, Chtara, Brugheli, Turki, Galy, Chamari \& Behm, 2010; LitTLE \& Williams, 2006). Algumas das principais justificativas são: semelhança dos padrões de movimento utilizados (NeEdham, Morse \& Degens, 2009), aumento da temperatura corporal (TAYLOR, Sheppard, Lee \& Plummer, 2009), auxílio na propriocepção e permissão de melhor pré-ativação do organismo para a tarefa posterior (GELEN, 2010).

Os achados mais gerais descritos pelos estudos realizados até agora direcionam para a perda no rendimento em corridas curtas (Siatras, Papadopoulos, Mameletzi, Gerodimos \& Kellis, 2003). Porém, frente à diversidade de informaçôes, é necessário considerar os diferentes elementos que compõem a realização dos testes. Além disso, o tipo de estímulo proporcionado pela prática estática ou dinâmica aponta para direçôes contrárias, uma vez que até então os resultados apontam para diferentes desfechos de acordo com a escolha de um ou de outro, e isso faz com que seja necessário analisar cada um dos estudos e tentar determinar, quantitativamente, o quanto de perda se pode ter com a realização das técnicas de alongamento, e mais do que isso é conseguir diferenciar os resultados de acordo com cada tipo de exercício executado antes da realização de CCAI/“sprints”, para qual se encontram pontos positivos e em qual estão os piores resultados, entre o estático e o dinâmico.

Portanto, o objetivo desse estudo foi examinar os efeitos de diferentes protocolos de alongamentos, realizados como componentes do aquecimento, anteriormente à execução de testes envolvendo CCAI/"sprints", utilizando duas ferramentas de análise, o Tamanho de Efeito (TE) e o Delta Percentual $(\Delta \%)$.

\section{Métodos}

A presente investigação se constitui como metanálise, a qual objetiva unir algum problema em comum de diferentes estudos, integrando-os para então analisá-los de maneira conjunta (HEDGES \& OLKIN, 1985). Com essa ferramenta é possível aumentar o tamanho da amostra e se permite descrição mais precisa e adequada dos efeitos do alongamento no desempenho de CCAI.

O desenvolvimento do trabalho ocorreu seguindo o esquema apresentado no QUADRO 1. 
QUADRO1-Fluxograma dos procedimentos para seleção dos artigos inseridos na análise durante o estudo.

Artigos retornados a partir de busca com palavraschave "sprint" e "stretching" nas bases de dados e potencialmente relevantes para o estudo $(n=20)$.

Número de estudos excluídos que apresentavam sexo feminino e idade inferior a 16 anos $(n=5)$.

Trabalhos excluídos da análise por não apresentarem procedimentos envolvendo situação sem alongamento $(n=4)$.

Trabalhos com dados originais, seres humanos, sem restriçôes de modalidades, nível de condicionamento e protocolo de avaliação foram incluídos para leiutra do texto completo $(n=15)$.

Número final de estudos incluídos na análise $(\mathrm{n}=11)$.

\section{Amostra}

A pesquisa foi realizada usando unicamente dois termos de referência em língua inglesa - "sprint" e "stretching" - os quais foram inseridos nas bases de dados PubMed, SPORTDiscus e EBSCO-host, com buscas feitas no período compreendido entre 23 de agosto e 16 de outubro de 2010, sem limites de data de publicação. Complementarmente, foram recuperadas e incorporadas, caso atendessem aos critérios de inclusão, as referências utilizadas nos artigos obtidos durante as buscas. Consideraram-se adequados os materiais publicados em jornais e revistas que possuíam dados originais, envolvendo exercícios de alongamentos para os membros inferiores, pessoas do sexo masculino e com idade superior a 16 anos, além de exibirem os valores na condição sem alongamento. Não houve restrição quanto à modalidade, ao nível de aptidão física e aos procedimentos de avaliação utilizados nos estudos que compuseram as amostras dos artigos estudados.

$\mathrm{O}$ desempenho em corridas curtas, avaliado a partir do tempo, foi considerado como desfecho principal para as $11(\mathrm{n}=11)$ investigaçôes selecionadas, as quais proporcionaram 62 situações de análises, dado que no interior de cada estudo houve separação de diferentes eventos como, por exemplo, alongamentos estático, dinâmico e misto.

\section{Procedimentos}

Os artigos selecionados foram classificados segundo a Escala PEDro, a qual é composta por 11 questôes que visam proporcionar medida de qualidade metodológica a estudos clínicos, pois se verifica a validade interna dos trabalhos, além de identificar se existe possibilidade de informações suficientes para haver interpretação a partir da estatística descrita dos mesmos (MAHER, Sherrington, Hebert, Moseley \& Elkins, 2003).

Além disso, assumindo a quantidade de fatores que estão relacionados aos resultados obtidos em cada estudo, foi necessária incorporação de alguns indicadores de caracterização geral. Assim, as variáveis foram agrupadas da seguinte forma:

a) Características dos indivíduos: composto pelas médias de idade, estatura, massa corporal, nível de aptidão (Atleta ou Não-Atleta) e modalidade (Coletivas, Individuais ou Mistas); 
b) Delineamento do estudo: diz respeito aos componentes da avaliação do desempenho individual durante o estudo e como esses elementos foram alocados nos trabalhos. É formado, fundamentalmente, por aquecimento, alongamento e protocolos de avaliação. Para facilitar o entendimento de como foram dispostos em cada um dos estudos, considerou-se o critério da quantidade de testes e em que momento foram desempenhados (se antes ou depois do alongamento e aquecimento) para a caracterização dos estudos. Com isso, foram classificados em três tipos: 1) Teste único após aquecimento e alongamento; 2) Teste único entre aquecimento e alongamento; e 3) Vários testes após aquecimento e alongamento;

c) Características dos protocolos de alongamento: esse ponto versa sobre os tipos (Estático, Dinâmico ou Misto), a duração (o menor e o maior tempo de estímulos encontrados foram de 20 s e 60 s, para os estáticos e mistos, dado que os dinâmicos são determinados, quase que em sua maioria, por distâncias) e intensidade dos estímulos executados, ao se atingir o ponto de desconforto doloroso ou maior amplitude de movimento/ restrição mecânica causada pelos movimentos;

d) Protocolo de avaliação do desempenho: objetivamente foram classificados como: 1) Corridas até 20 metros; 2) Corridas acima de 20 metros; 3) Corridas múltiplas; e 4) Corridas com mudança de direção. Nenhum dos estudos foi alocado em mais de uma das características acima mencionadas.

A partir dos 11 trabalhos investigados para este estudo, indica-se que tempo aproximado entre o fim dos protocolos de alongamento e a avaliação para cinco dos textos foi nulo, ou seja, imediatamente após os estímulos eram realizadas as avaliaçōes e, para os outros seis, o tempo até o teste esteve entre um e sete minutos. Isso foi determinado a partir da leitura dos procedimentos, dos delineamentos experimentais dos estudos e com base nas suas figuras apresentadas.

\section{Análise estatística}

Para apresentação dos dados descritivos, foram utilizadas Média \pm EP e distribuição de frequências. Calcularam-se: 1) tamanho de efeito (TE, equação

\section{Resultados}

De modo geral, elevada quantidade de estudos (90,3\%) atinge sete pontos na Escala de PEDro, os demais chegaram a cinco pontos, do total de 11 possíveis. As características dos sujeitos
1), medida síntese para os diferentes estudos, que permite a verificação e interpretação de alterações ocorridas em diferentes estudos envolvendo vários grupos (RHEA, 2004), ou, em outros termos, pode ser denominada como a medida da magnitude das diferenças ocorridas entre os grupos, ou a condição experimental em relação à controle (THOMAs \& FRENCH, 1986); e 2) delta de variação ( $\Delta \%)$, medida que apresenta, em termos percentuais, a modificação no rendimento físico (equação 2 ).

Equação 1:

Tamanho do Efeito = Média Pós-teste - Média Pré-teste Desvio Padrão da situação Pré-teste

\section{Equação 2:}

Delta Percentual = 100·[(Valor Pré-teste/Valor Pós-teste) -1$]$

Para as comparações, foram realizadas duas análises de variância, a partir do comando "General Linear Model Univariate (GLM)" do "software" SPSS (v.16.0.0). Como condição para aplicação deste procedimento, previamente foram verificadas as homogeneidades das variâncias mediante o teste de Levene $(F=1,47, p=0,147)$, o qual indicou que os dados exibem igualdade de variância e podem ser testados com o respectivo GLM (FIELD, 2005).

Assim, o TE e o $\Delta \%$ foram considerados variáveis dependentes e, como fatores: Tratamento (Estático, Dinâmico, Misto), Delineamento (teste único após aquecimento e alongamento; teste único entre aquecimento e alongamento; vários testes), Número de séries (1, 2 ou 3 séries), Protocolo de avaliação empregado (até $20 \mathrm{~m}$, acima de $20 \mathrm{~m}$, RSA, corridas com mudança de direção), Prática de alongamento (presente ou não descrita) e Nível de Aptidão Física (amador ou atleta). Empregou-se "post-hoc" de Bonferroni para se identificarem os efeitos principais e suas interações (com exibição do poder observado), bem como no processo das comparações múltiplas (Maia, Garganta, Seabra, Lopes, Prista \& Freitas, 2004). Ainda, massa corporal, estatura, índice de massa corporal (IMC), idade e modalidade praticada foram posicionados como covariáveis.

envolvidos nestes estudos estão descritas na TABELA 1.

A frequência de ocorrência de cada variável analisada é exibida na TABELA 2. 
TABELA 1 - Descrição geral das características dos sujeitos dos estudos, através de Média 土EP e Amplitude.

\begin{tabular}{lcccc}
\hline Características dos Sujeitos & Média & EP & Mínimo & Máximo \\
\hline Idade (anos) & 21,40 & 0,18 & 16,8 & 24 \\
Massa corporal (kg) & 73,90 & 0,54 & 68,2 & 86,5 \\
Estatura (cm) & 179,94 & 0,32 & 171,0 & 185,7 \\
IMC & 22,93 & 0,12 & 22,34 & 26,40 \\
\hline
\end{tabular}

TABELA 2 - Frequências absoluta e relativa das variáveis selecionadas para análise.

\begin{tabular}{|c|c|c|}
\hline Variáveis consideradas & Frequência Absoluta & Frequência Relativa (\%) \\
\hline \multicolumn{3}{|l|}{ Escala de PEDro } \\
\hline 5 pontos & 06 & 09,7 \\
\hline 7 pontos & 56 & 90,3 \\
\hline Total & 62 & 100 \\
\hline \multicolumn{3}{|l|}{ Prática de alongamento } \\
\hline Sim & 28 & 45,2 \\
\hline Não relatado & 34 & 54,8 \\
\hline Total & 62 & 100 \\
\hline \multicolumn{3}{|l|}{ Nível de aptidão } \\
\hline Atletas & 59 & 95,2 \\
\hline Amadores & 03 & 04,8 \\
\hline Total & 62 & 100 \\
\hline \multicolumn{3}{|l|}{ Modalidade } \\
\hline Coletivas & 28 & 45,2 \\
\hline Individuais & 06 & 09,6 \\
\hline Mistas & 28 & 45,2 \\
\hline Total & 62 & 100 \\
\hline \multicolumn{3}{|l|}{ Delineamento do Estudo } \\
\hline Teste único após aquecimento e alongamento & 14 & 22,6 \\
\hline Teste único entre aquecimento e alongamento & 08 & 12,9 \\
\hline Vários testes & 40 & 64,5 \\
\hline Total & 62 & 100 \\
\hline \multicolumn{3}{|l|}{ Tipo de Alongamento } \\
\hline Estático & 29 & 46,8 \\
\hline Dinâmico & 12 & 19,3 \\
\hline Misto e outros & 21 & 33,9 \\
\hline Total & 62 & 100 \\
\hline \multicolumn{3}{|l|}{ Protocolo de Avaliação } \\
\hline Corridas até 20 metros & 17 & 27,4 \\
\hline Corridas acima de 20 metros & 22 & 35,5 \\
\hline Corridas Múltiplas & 07 & 11,3 \\
\hline Corridas com mudança de direção & 16 & 25,8 \\
\hline Total & 62 & 100 \\
\hline
\end{tabular}

Continua
*Apenas um dos estudos selecionados não disponibilizou os valores referentes às características descritas nesta tabela.

Rev. bras. Educ. Fís. Esporte, São Paulo, v.25, n.4, p.567-81, out./ dez. 2011 • 571 
TABELA 2 - Frequências absoluta e relativa das variáveis selecionadas para análise (continuação).

\begin{tabular}{lcc}
\hline Variáveis consideradas & Frequência Absoluta & Frequência Relativa (\%) \\
\hline Número de Séries & 17 & 27,4 \\
Única & 39 & 62,9 \\
Duas & 06 & 09,7 \\
Três & 62 & 100 \\
$\quad$ Total & & \\
\hline Sentido do Tamanho de Efeito & 39 & 62,9 \\
Negativo & 20 & 32,3 \\
Positivo & 03 & 04,8 \\
Nulo & 62 & 100 \\
$\quad$ Total & & \\
\hline Sentido do Delta Percentual & 39 & 62,9 \\
Negativo & 39 & 32,3 \\
Positivo & 20 & 04,8 \\
Nulo & 03 & 100 \\
$\quad$ Total & 62 & \\
\hline
\end{tabular}

Observa-se que mais da metade $(54,8 \%)$ das situaçōes encontradas neste estudo não relataram se os sujeitos analisados realizavam práticas de alongamento em suas sessões cotidianas de treinamento. Além disso, grande maioria $(95,2 \%)$ dos avaliados nos artigos selecionados é composta por atletas. Já para as modalidades praticadas, foi encontrado mesmo percentual $(45,2 \%)$ para as coletivas e mistas (coletivas e individuais), e 9,6\% de modalidades individuais.

No que diz respeito ao delineamento do estudo, em $64,5 \%$ das investigações ocorreram avaliações com diversos testes (corridas e saltos), nas quais $35,5 \%$, $27,4 \%$ e $25,8 \%$ foram de corridas com distância superior a $20 \mathrm{~m}$, corridas de até $20 \mathrm{~m}$ e corridas com mudança de direção, respectivamente. Para os tipos de alongamento, $46,8 \%$ das situações fizeram uso do procedimento executado de modo estático, 33,9\% foram com práticas mistas e outros e $19,3 \%$ foram com alongamentos dinâmicos. Duas séries de estímulos de alongamento foi o número que se apresentou em maior percentagem, com $62,9 \%$, sendo que a realização de série única foi responsável por $27,4 \%$ dos experimentos e três séries foram observadas em 9,7\% deles.

Os valores percentuais do sentido negativo do TE e do $\Delta \%$ perfazem $62,9 \%$, e representa que 39 das 62 situações estudadas proporcionaram efeitos prejudiciais no desempenho em testes subsequentes. O sentido positivo do TE e do $\Delta \%$ foi observado em $32,3 \%$ dos casos, e efeito nulo em $4,8 \%$.

A análise realizada apresentou como resultado para o Tamanho de Efeito (TE) valor médio de -0,06 $( \pm 0,06)$, com amplitude entre $-1,95$ e 1,50 para as investigaçôes incluídas neste estudo. Além disso, para o $\Delta \%$, obteve-se média de $-0,41 \%( \pm 0,26)$ com amplitude entre $-7,85 \%$ e $5,42 \%$.

Observa-se, na TABELA 3, que o delineamento de mensuração adotado pelos pesquisadores apresenta resultados estatisticamente diferentes quando da realização de um único teste após alongamento e aquecimento em comparação à execução de vários testes $(\mathrm{F}=14,97 ; \mathrm{p}<0,001)$. Além disso, o tipo de alongamento afeta o desempenho em CCAI, com o alongamento dinâmico sendo diferente dos demais, tanto para o TE $(\mathrm{F}=33,14 ; \mathrm{p}<0,001)$, quanto para o $\Delta \%(\mathrm{~F}=34,84 ; \mathrm{p}<0,001)$. Ainda, o protocolo de avaliação também proporciona diferenças significantes $(\mathrm{F}=7,75 ; \mathrm{p}=0,05)$, as quais são observadas entre corridas com mudanças de direção e corridas lineares únicas, mas isto só é observado no TE. Por fim, estímulos de alongamento com séries únicas se mostraram ser menos prejudiciais frente àqueles que fazem uso de séries sucessivas $(\mathrm{F}=19,83 ; \mathrm{p}<0,001)$.

Em referência ao delta percentual, a FIGURA 1 apresenta os gráficos das variáveis que exibiram diferenças significantes quanto aos procedimentos adotados.

Por fim, são observadas interações entre alguns fatores: 1) execução de alongamentos estáticos ( $\mathrm{p}=$ 0,001 , poder $=0,99$ ) ou de alongamentos dinâmicos $(\mathrm{p}=0,03$, poder $=0,57) \mathrm{com}$ rotina pregressa $\mathrm{e}$ experiência na prática de alongamentos; 2) Execução de série única com o tipo de alongamento estático $(\mathrm{p}=0,02$, poder $=0,66)$ e dinâmico $(\mathrm{p}=0,001$, poder $=0,99)$. 
TABELA 3 - Medidas descritivas (média Łep), valor de F e nível de significância do tamanho do efeito e do delta percentual, segundo diferentes variáveis consideradas.

\begin{tabular}{|c|c|c|c|c|c|c|c|}
\hline Variáveis & TE & $\mathbf{F}$ & $\mathbf{P}$ & $\Delta \%$ & $\mathbf{F}$ & $\mathbf{P}$ & \multirow{10}{*}{$\begin{array}{l}\text { *Apresenta diferença } \\
\text { significativa entre os } \\
\text { grupos. } \\
\dagger \text { Diferença significativa } \\
\text { para com a realizaçãc } \\
\text { de vários testes ( } p= \\
0,001) \text {. } \\
\text { † Diferença significativa } \\
\text { para os grupos Estáticc } \\
(p<0,001) \text { e Misto } \\
\text { outros }(p<0,02) \text {. } \\
\text { † Diferença significativa } \\
\text { para corridas até } 20 \mathrm{~m} \\
(p=0,003) \text { e corridas } \\
\text { acima de } 20 \mathrm{~m}(p \\
0,009) . \\
\text { \# Diferença significativa } \\
\text { para duas }(p=0,016) \text { e } \\
\text { três }(p<0,001) \text { séries. }\end{array}$} \\
\hline Delineamento* & & 14,97 & 0,001 & & 4,84 & 0,015 & \\
\hline $\begin{array}{l}\text { Teste único após aquecimento e } \\
\text { alongamento } \dagger\end{array}$ & $-0,20 \pm 0,1$ & & & $-0,90 \pm 0,28$ & & & \\
\hline $\begin{array}{l}\text { Teste único entre aquecimento e } \\
\text { alongamento }\end{array}$ & $-0,03 \pm 0,08$ & & & $-0,34 \pm 0,16$ & & & \\
\hline Vários testes & $0,01 \pm 0,1$ & & & $0,22 \pm 0,23$ & & & \\
\hline Tipo de alongamento* & & 33,14 & $<0,001$ & & 34,84 & 0,001 & \\
\hline Estático & $0,20 \pm 0,1$ & & & $-0,16 \pm 0,19$ & & & \\
\hline Dinâmicođ & $0,46 \pm 0,1$ & & & $1,85 \pm 0,25$ & & & \\
\hline Misto e outros & $-0,17 \pm 0,07$ & & & $-0,13 \pm 0,24$ & & & \\
\hline Modalidade & & 2,11 & 0,130 & & 2,41 & 0,098 & \\
\hline Coletivas & $0,07 \pm 0,1$ & & & $0,12 \pm 0,4$ & & & \\
\hline Individuais & $-0,23 \pm 0,03$ & & & $-1,33 \pm 0,4$ & & & \\
\hline Mistas & $-0,18 \pm 0,05$ & & & $-0,85 \pm 0,2$ & & & \\
\hline
\end{tabular}

\section{Prática de alongamento}

$\begin{array}{cccc}2,54 \quad 0,123 & 2,06 & 0,156 \\ & -0,85 \pm 0,2 & & \\ & -0,11 \pm 0,4 & & \end{array}$

$\operatorname{Sim}$

$-0,18 \pm 0,05$

$-0,11 \pm 0,4$

\section{Nível de Aptidão}

Profissionais

Amadores

$$
0,02 \pm 0,1
$$

0,852

$0,20 \quad 0,655$

$-0,07 \pm 0,07$

$-0,46 \pm 0,2$

$-0,02 \pm 0,1$

\begin{tabular}{|c|c|c|c|c|c|c|}
\hline Protocolo de Avaliação* & & 7,75 & 0,05 & & 1,27 & 0,291 \\
\hline Corridas até 20 metros & $-0,16 \pm 0,1$ & & & $-1,02 \pm 0,3$ & & \\
\hline Corridas acima de 20 metros & $-0,12 \pm 0,1$ & & & $-0,58 \pm 0,4$ & & \\
\hline Corridas múltiplas & $-0,05 \pm 0,1$ & & & $-0,12 \pm 0,4$ & & \\
\hline Corridas com mudança de direção $\ddagger$ & $0,10 \pm 0,1$ & & & $0,31 \pm 0,6$ & & \\
\hline Número de Séries* & & 19,83 & $<0,001$ & & 6,52 & 0,01 \\
\hline Única\# & $0,12 \pm 0,1$ & & & $0,20 \pm 0,22$ & & \\
\hline Duas & $-0,13 \pm 0,08$ & & & $-0,065 \pm 0,16$ & & \\
\hline Três & $-0,23 \pm 0,03$ & & & $-1,33 \pm 0,34$ & & \\
\hline
\end{tabular}


A

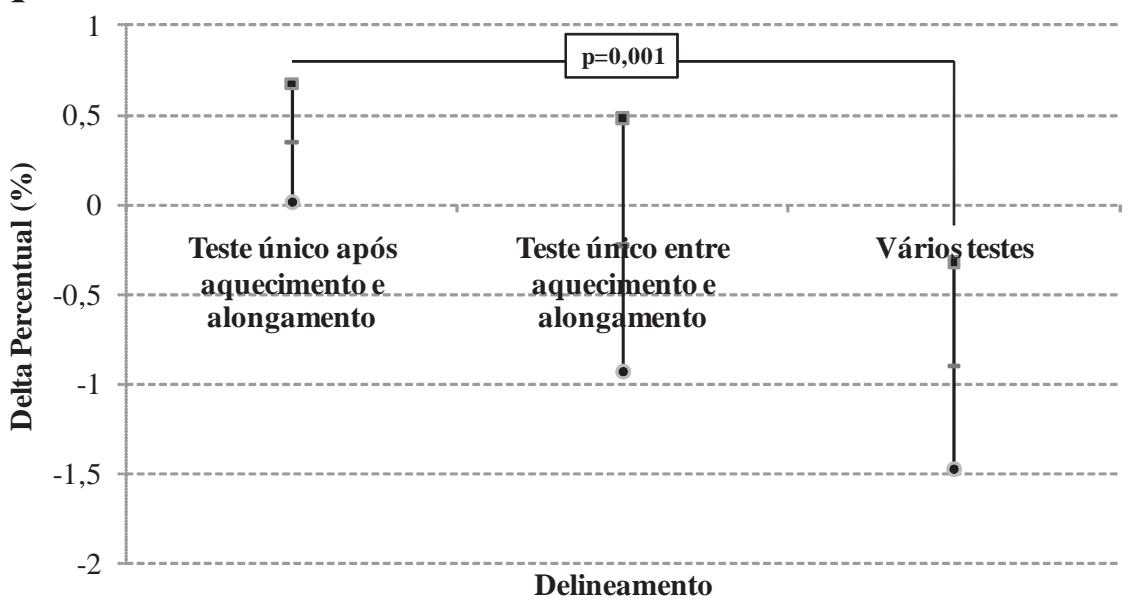

B

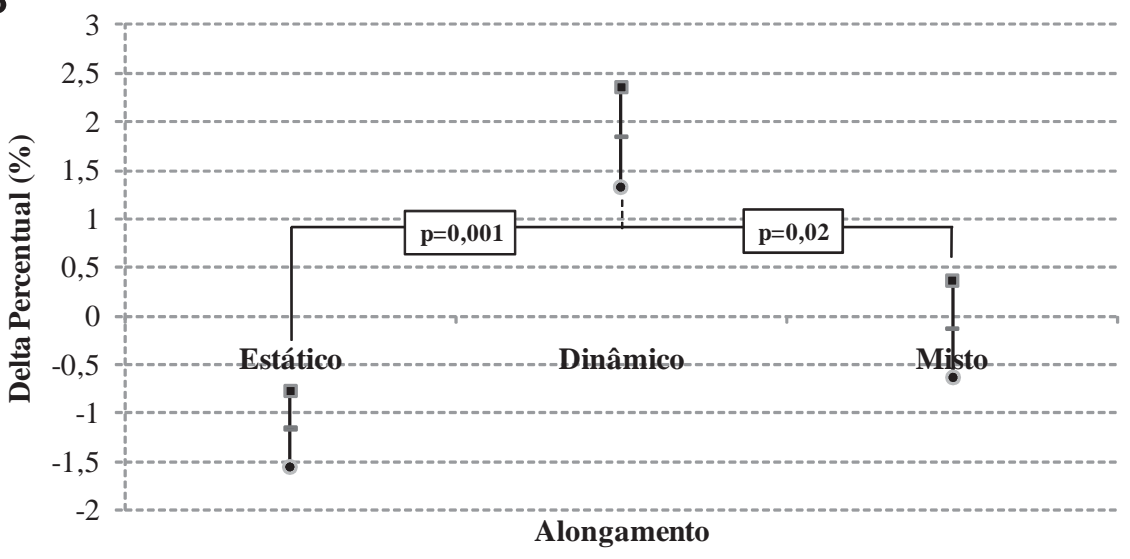

C

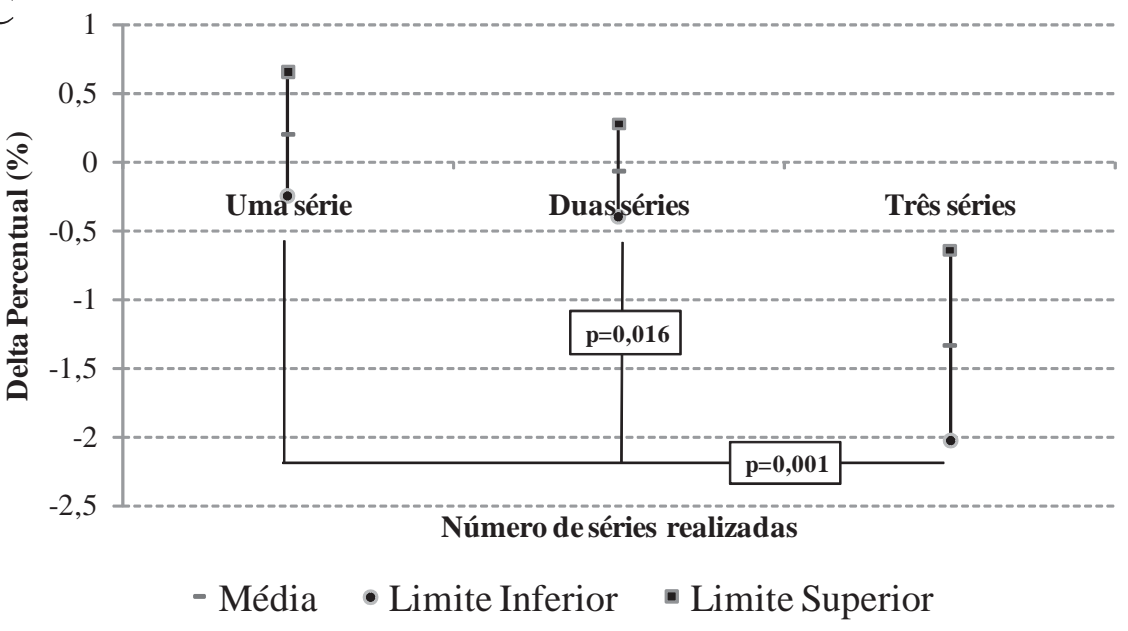

FIGURA 1 - Média elimites superior e inferior do intervalo de confiança (95\%) referentes ao delta percentual das variáveis de interesse. As informações sobre tipo de delineamento, procedimento de alongamento e número de séries estão apresentadas, respectivamente, nos gráficos A, B e C. 


\section{Discussão}

Como o objetivo do presente estudo foi verificar a magnitude da utilização dos protocolos de alongamentos em subsequentes desempenhos em CCAI, o principal achado foi que diferentes modos alongamento proporcionam efeitos específicos. Neste contexto, identificou-se que práticas dinâmicas promovem melhor rendimento no desempenho de CCAI, tanto para o TE, quanto para o $\Delta \%$, quando comparadas com técnicas estáticas $(\mathrm{p}<0,001)$ ou mistas $(\mathrm{p}<0,01)$. Além disso, dentre os procedimentos existe diferença na execução de um único teste após alongamento e aquecimento frente à realização de vários testes $(\mathrm{p}=$ $0,001)$. Quanto ao protocolo de avaliação, há diferença entre corridas com mudanças de direção e corridas lineares até $20 \mathrm{~m}(\mathrm{p}=0,003)$ e corridas com mudança de direção e corridas lineares acima de $20 \mathrm{~m}(\mathrm{p}<0,009)$, mas isto só é observado no TE. Por fim, protocolos de alongamento com séries únicas podem ter menor interferência quando comparados a estímulos com duas $(\mathrm{p}=0,016)$ e três séries $(\mathrm{p}<0,001)$.

Evidências recentes (ChaOUachi et al., 2010; Gelen, 2010; Little \& Williams, 2006; Needham, Morse \& Degens, 2009; Taylor et al., 2009) corroboram com os achados deste estudo, os quais sugerem que alongamentos dinâmicos $(\mathrm{AD})$ são benéficos para o rendimento posterior em CCAI. As justificativas estão no fato de a estimulação dinâmica (MuRPHY, 1994) aumentar a temperatura do tecido muscular e, assim, elevar: a) a eficácia da contração (Martin, Robinson, Wiegman \& Aulick, 1975); b) a velocidade de transmissão de impulsos nervosos; e c) o trabalho muscular (SAFRAN, SAEber \& GARRETt Junior, 1989). Além disso, são movimentos que podem se assemelhar à tarefa a ser executada e, por conseguinte, elevar a excitabilidade das unidades motoras e a capacidade de produção de potência (Faigenbaum, Kang, McFarland, BloOm \& Magnatta, 2006; Faigenbaum, McFarland, Schwerdtman, Ratamess, Kang \& Hoffman, 2006). O AD proporcionou melhores resultados em estudos com jogadores profissionais de futebol, com diferenças significantes entre fazer alongamentos dinâmico, estático e não alongar para a execução subsequente de CCAI de 10 a 30 metros e para testes de agilidade (Amiri-Khorasani et al., 2010; Gelen, 2010; Little \& Williams, 2006). Já com estudantes altamente treinados em diferentes modalidades, não foram identificadas alterações no resultado de CCAI nas distâncias de cinco a 30 metros, tampouco em teste de agilidade para as práticas de $\mathrm{AD}$, AE e AM (CHAOUACHI et al., 2010). É possível que os resultados acima mencionados tenham sido encontrados em função de o grupo de jogadores de futebol apresentar média etária e tempo de experiência maior que os estudantes treinados. Porém, não se infere que o tipo e as características do treinamento possam ser prováveis fatores de confusão para esses resultados (AMIRI-KHORASANI et al., 2010).

Diferente dos estudos que envolvem $\mathrm{AD}$, os resultados para os experimentos que praticam $\mathrm{AE}$ mostram redução do desempenho em subsequentes CCAI (Amiri-Khorasani et al., 2010; Fletcher \& AnNess, 2007; Fletcher \& Jones, 2004; Kistler et al., 2010; WinCHESTER et al., 2008), o que reforça os resultados da presente investigação para o TE e $\Delta \%$. A grande maioria deles relaciona a perda de rendimento ao fato de que o $\mathrm{AE}$ pode influenciar em elementos periféricos e/ou neurais. Entretanto, é preciso deixar explícito que essas explicaçóes, na tentativa de entender o que acontece durante o procedimento de alongar e posteriormente verificar o resultado em CCAI, são sugeridas a partir de estudos que utilizam procedimentos laboratoriais e não eventos de campo, o que aumentaria confiabilidade/reprodutibilidade, mas diminuiria a validade ecológica dos mesmos (Reilly, Morris \& White, 2009). Seria interessante que simulações da prática em seu contexto real pudessem contar com avaliação do desempenho nas situações esportivas específicas de cada modalidade. Portanto, é necessário cuidado com a interpretação e transferência dos resultados obtidos, sendo assim, também com as justificativas e explicações sugeridas.

No que diz respeito aos ajustes periféricos, o prejuízo do AE deriva de modificações das estruturas da unidade músculotendínea (UMT) (McNair \& Stanley, 1996), pois a rigidez de seus componentes pode aumentar a produção de força dos componentes contráteis devido ao aumento do comprimento e frequência de encurtamento (Wilson, Murphy \& PrYor, 1994).

Para STONE et al. (2006), a rigidez tecidual (em inglês, denominada "stiffness") é a capacidade de o tecido resistir às mudanças no seu comprimento e é representada pela divisão das alterações de força pelas alteraçōes de seu comprimento $(\Delta \mathrm{F} / \Delta \mathrm{C})$. Sendo assim, a realização do $\mathrm{AE}$ promoveria redução do grau de rigidez da UMT, o que prejudicaria o rendimento durante o movimento. A perda desta rigidez pode ter relação com a diminuição da resistência passiva ao alongamento. Além disso, McHugh e Cosgrave 
(2010) relatam que o incremento da propriedade viscoelástica, gerado pelo $\mathrm{AE}$, eleva a amplitude de movimento e diminui a resistência ao alongamento.

Somado a esses fatores existe o argumento que relaciona a queda de rendimento em CCAI à diminuição da capacidade dos componentes elásticos estocarem energia, para que a mesma seja utilizada no ciclo de alongamento-encurtamento durante exercícios que necessitam de produção de potência e taxa de desenvolvimento de força, a partir da UMT (Shorten, 1987). Todavia, apesar de todas essas sugestões, Morse, Degens, Seynnes, Maganaris e JONES (2008) relatam que as propriedades tendíneas "in vivo" permanecem inalteradas após o AE. Para sustentar esse achado, há estudos nos quais protocolos de intervenção com alongamentos que duraram menos de quatro minutos provocaram perda de força, mas esta queda não esteve ligada à diminuição da rigidez muscular (KOKKONEN, NELSON \& CORNWELL, 1998; McNair \& Stanley, 1996; Nelson, Driscoll, Landin, Young \& Schexnayder, 2005;Sekir, Arabaci, AKova \& Kadagan, 2010).

Diante dessa falta de consenso na literatura, é possível que a outra perspectiva venha a complementar a explicação para tais achados. A justificativa que trata dos efeitos neurais se sustenta no fato de que os exercícios de alongamento impedem adequada potencialização mioelétrica (PM) das capacidades que envolvem o ciclo de alongamento-encurtamento (CAE). Isso porque Bosco, VitTasalo, Komi e LuHTANEN (1982) propuseram que a fase excêntrica do CAE inicia com tal potencialização, a qual resulta em estiramento reflexo (ER), proporcionando aumento da ativação muscular durante o período de trabalho concêntrico. $\mathrm{O}$ incremento da capacidade de ceder promovido pelo $\mathrm{AE}$ pode resultar em menor ativação dos fusos musculares na fase excêntrica do movimento, o que afetaria negativamente o estágio concêntrico (KISTLER et al., 2010). Adicionalmente, Rosenbaum e Hennig (1995) relataram que o alongamento muscular pode diminuir a força do estiramento reflexo, observado no tendão do calcâneo, o que prejudicaria o CAE.

Mecanismos centrais (sistema nervoso central e periférico) parecem ser responsáveis pela alteração desta atividade reflexa, como menor excitabilidade neuromotora decorrente dos estímulos de alongamento (Avela, Kyrolainen \& Komi, 1999; Guissard, Duchateau \& Hainaut, 1988), além da sugestão de que estão ligados aos eventos pré e pós-sinápticos. As modificaçōes pré-sinápticas que podem explicar a queda do reflexo proprioceptivo, reflexo de Hoffman $(\mathrm{H})$, quando em alongamentos tratam sobre: a) diminuição autogênica de uma das vias aferentes, a qual é induzida pela inibição pré-sináptica (DelWAide, 1973; Rudomin, 1990); b) capacidade de transmissão sináptica durante ativação repetida (Crone \& Nielsen, 1989; KoHN, Floeter \& Hallett, 1997). Já as mudanças póssinápticas que justificam o déficit na atividade reflexa $\mathrm{H}$ indicam: a) inibição autogênica induzida pelos órgãos tendinosos de Golgi (HOUK, CRAG \& RYMER, 1980; Robinson, McComas \& Belanger, 1982); b) inibição a partir dos receptores aferentes articulares e cutâneos (CACCIA, McComas, Upton \& BlogG, 1973; LundBerg, MaLmgren \& SCHOMburG, 1978); c) que a via aferente supraespinhal pode ter atuação prejudical no reflexo espinhal durante o alongamento (Guissard, Duchateau \& Hainaut, 2001).

A combinação entre $\mathrm{AD}$ e $\mathrm{AE}$, denominado alongamento misto (AM), parece não promover melhora no desempenho quando comparado exclusivamente com a prática do $\mathrm{AD}$; entretanto é possível que o $\mathrm{AM}$ produza melhores resultado em relação ao $\mathrm{AE}$, já que em situaçóes de CCAI de 10 a 30 metros e testes de agilidade com jogadores de futebol foi detectado esse fato (Amiri-Khorasani et al., 2010; Gelen, 2010; Little \& Williams, 2006). Por outro lado, com estudantes treinados em diferentes modalidades, não foram identificadas alterações no resultado de CCAI nas distâncias de cinco a 30 metros, tampouco em teste de agilidade, tanto de maneira isolada, quanto com combinaçōes variadas de $\mathrm{AD}, \mathrm{AE}$ e $\mathrm{AM}$ em diferentes intensidades (CHAOUACHI et al., 2010).

Nos delineamentos, a realização de ordens diferentes de avaliação pode ser fator de interferência no resultado das investigações e se identificou de determinada disposição dos procedimentos pode alterar o rendimento, dada a observância de diferença para dois dos três tipos de delineamentos, a saber: a) teste único após o alongamento e aquecimento (AMIRI-KHORASANI et al., 2010; WinCHESTER et al., 2008); e b) investigações que aplicam diferentes testes em sequência após os estímulos de alongamento e aquecimento (CHAOUACHI et al., 2010; GeLEN, 2010), sendo que este último delineamento proporcionou melhores resultados nas avaliações. Neste contexto, cogita-se que os sujeitos já apresentassem elevação de temperatura corporal e, consequentemente muscular, além de ativação e predisposição que permitiria melhor rendimento na tarefa subsequente (BISHOP, 2003).

Quanto ao número de séries, havia possibilidade de se encontrarem diferenças entre os protocolos, ou seja, quanto mais séries, maior efeito agudo negativo no rendimento. Isso se dá em virtude do 
tempo total de estímulo, pois os menores e maiores tempos de estímulo foram, respectivamente, de 20 s e $60 \mathrm{~s}$; além disso, grande parte dos protocolos estabelecia que a intensidade estivesse em torno do ponto de desconforto ou maior amplitude de movimento (ChaOUachi et al., 2010; Favero, Midgley \& Bentley, 2009; Little \& Williams, 2006; Wong, Lau, Mao, Wu, Behm \& Wisloff, 2011). De acordo com os resultados do presente estudo, foi confirmada essa hipótese, ao se comparar realização de série única com duas e três séries. Segundo McHugh e Cosgrave (2010), os efeitos de quatro minutos totais de duração de alongamento ainda podem ser observados 10 minutos após o estímulo, sem atividade/exercício posterior. Logo, com este tempo total mínimo (de quatro minutos), as práticas de alongamento ainda promoveriam algum efeito deletério residual após utilização de $\mathrm{AE}$, o que parece ter ocorrido nos estudos utilizados, pois a série única não atingiria tempo total de quatro minutos de estímulo, diferente das séries sucessivas.

Para os protocolos de avaliação, foi confirmado que há diferença entre os tipos de avaliações do desempenho, porém não com os protocolos esperados, já que houve diferença entre corridas com mudança de direção e corridas lineares (até 20 metros e para as acima de 20 metros), o que é contrário aos achados de FaIGENBAUM, Bellucci, Bemieri, Bakker e Hoorens (2005), no qual não foram encontradas diferenças estatísticas em avaliações com mudança de direção. Esperava-se que fosse possível existir diferença entre corridas múltiplas e as demais, em função de a repetição da atividade minimizar ou eliminar algum efeito deletério que o alongamento proporcionasse (BECKETT et al., 2009).

Uma das limitações do estudo foi a não consideração dos valores das situações controle como fator protocolo nas análises de variância. Isto se deu por dois motivos. O primeiro é a inclusão deste dado no cálculo do tamanho do efeito (RHeA, 2004) e do delta percentual. O segundo reside no fato de que os estudos não apresentam situação placebo, para controle verdadeiro, ou seja, a medida de controle é feita antes da execução dos procedimentos de intervenção, o que em parte reflete a baixa pontuação dos estudos na escala PEDro. Complementarmente, apenas a seleção de estudos que a amostra era composta pelo sexo masculino pode ter limitado a quantidade de observaçôes; no entanto, investigações com mulheres não têm controlado o perfil hormonal e ciclo menstrual das mesmas, o que dificulta as inferências (MIDDLETON \& Wenger, 2006). Por fim, outra limitação reside no fato de alguns artigos não apresentarem valores exatos das avaliaçôes de rendimento, dado que eram exibidos em gráficos (por exemplo, LiTTLE \& Williams, 2006).

Baseados nos resultados apresentados, há situação de reflexão: o que apresenta diferenças perante análises estatística e o que, de fato, é efetivo ou significante para situações práticas/clínicas (STAPLETON, SCOTT \& AtKinson, 2009). Ainda não é possível realizar avaliações que apresentem condições de simulação idênticas à realidade e, juntamente a isto, pode-se considerar que cada situação tem suas características próprias e peculiaridades, dificultando o processo de translação do conhecimento. Portanto, o que determina a transferência de achados como o da presente investigação é a habilidade de interpretação de acordo com a prática de cada treinador/professor, ao se realizar análise adequada e com fundamentos suficientes para comparação das situaçōes e, então, fazer o uso adequado dos dados presentes na literatura.

\section{Conclusão}

Os achados da presente investigação sugerem que: 1) a realização de $\mathrm{AD}$ proporciona resultado benéfico quando comparado às práticas que envolvem $\mathrm{AE}$ ou $\mathrm{AM} ; 2$ ) pode existir diferença entre os delineamentos adotados quando da avaliação de efeitos de alongamentos em atividades subsequentes; 3) o tipo de protocolo de avaliação do rendimento no estudo também pode produzir resultados distintos; e 4) uma maior quantidade de séries de estímulos de alongamento pode provocar efeito negativos no desempenho. Não foram encontradas diferenças estatisticamente significantes para o desempenho em CCAI considerando o nível de aptidão dos sujeitos, sua modalidade praticada ou a prática pregressa de alongamentos. Sugere-se que os novos estudos indiquem a experiência dos sujeitos quanto à frequência, intensidade e tipo de alongamento que executam em suas rotinas de treinamento, pois vivências diferenciadas podem proporcionar respostas orgânicas variadas, no sentido de pessoas mais experientes em realizar alongamentos dinâmicos não terem decréscimos significativos nos testes de CCAI. 


\begin{abstract}
Meta-analysis of the acute effects of stretching on high intensity sprint performance

The literature on the effects of stretching on sprint performance is controversial. This meta-analysis aimed at examining the outcomes of the studies using different stretching protocols prior to sprint efforts. The search was conducted in several databases, using combinations of the following keywords: sprinting and stretching. Inclusion criteria included studies with males aged over 16 years irrespective of the sport, fitness level and assessment procedures performed. After the evaluation of the selected studies, 11 were considered appropriate for the analysis, resulting in 62 cases to be studied. The effect size (ES) and delta percentage $(\Delta \%)$ were considered as dependent variables. The study design, type of stretching, assessment protocol, number of sets, sport, fitness level and previous practice of stretching were considered as factors. The results suggest that: a) dynamic stretching (DS) significantly improves performance when compared to static (SS) $(p<0.001)$ or mixed (MS) $(p<0.002)$ stretching methods; $b)$ there are significant differences in $\mathrm{ES}$ and $\Delta \%$ between runs with change of direction and linear races (up to $20 \mathrm{~m}, \mathrm{p}=0.003$, and above $20 \mathrm{~m}, \mathrm{p}<0.009$ ); $\mathrm{c}$ ) performing multiple tests provides better results than a single test after the warm-up and stretch $(p=0.001)$, and $d)$ performing a single bout of stretching is less harmful to performance than two $(p=0.016)$ and three $(p<0.001)$ bouts. Therefore, it is possible to obtain small gains in high-intensity sprinting performance by using DS when compared with SS, MS or no stimuli.
\end{abstract}

UnITERMs: Stretching; High intensity sprint; Acute effect; Physical Performance.

\title{
Referências
}

AMIRI-KHORASANI, M.; SAHEBOZAMANI, M.; TABRIZI, K.G.; YUSOF, A.B. Acute effect of different stretching methods on Illionois Agility Test in soccer players. Journal of Strength and Conditioning Research, Champaign, v.24, n.10, p.2698-704, 2010. AVELA, J.; KYROLAINEN, H.; KOMI, P.V. Altered reflex sensitivity after repeated and prolonged passive muscle stretching. Journal of Applyed Physiology, Bethesda, v.86, p.1283-91, 1999.

BECKETT, J.R.; SCHNEIKER, K.T.; WALLMAN, K.E.; DAWSON, B.T.; GUELFI, K.J. Effects of static stretching on repeated sprint and change of direction performance. Medicine and Science in Sports and Exercise, Madison, v.41, n.2, p.444-50, 2009. BISHOP, D. Warm up II: performance changes following active warm up and how to structure the warm up. Sports Medice, Auckland, v.33, n.7, p.483-98, 2003.

BOSCO, C.; VIITASALO, J.T.; KOMI, P.V; LUHTANEN, P. Combined effect of elastic energy and myoelectrical potentiation during stretch-shortening cycle exercise. Acta Physiological Scandinavica, Oxford, v.114, p.557-65, 1982. CACCIA, M.R.; McCOMAS A.J.; UPTON A.; BLOGG T. Cutaneous reflexes in small muscles of the hand. Journal of Neurology, Neurosurgery, and Psychiatry, London, v.36, p.960-77, 1973.

CHAOUACHI, A.; CASTAGNA, C.; CHTARA, M.; BRUGHELli, M.; TURKI, O.; GALY, O.; CHAMARI, K.; BEHM, D.G. Effect of warm-ups involving static or dynamic stretching on agility, sprinting, and jumping performance in trained individuals. Journal of Strength and Conditioning Research, Champaign, v.24, n.8, p.2001-11, 2010.

CRONE C.; NIELSEN J. Methodological implications of the post activation depression of the soleus H-reflex in man. Experimental Brain Research, Berlin, v.78, p.28-32, 1989.

DELWAIDE, P.J. Human monosynaptic reflexes and presynaptic inhibition: an interpretation of spastic hyperreflexia. In: DESMEDT, J.E. (Ed). New developments in electromyography and clinical neurophysiology. Basel: Karger, 1973. v.3, p.508-22. de VILLARREAL, E.S; KELLIS, E.; KRAEMER, W.J.; IZQUIERDO, M. Determining variables of plyometric training for improving vertical jump height performance: a meta-analysis. Journal of Strength and Conditioning Research, Champaign, v.23, p.495-506, 2009.

FAIGENBAUM, A.D.; BELLUCCI, M.; BEMIERI, A.; BAKKER, B.; HOORENS, K. Acute effects of different warm-up protocols on fitness performance in children. Journal of Strength and Conditioning Research, Champaign, v.19, p.376-81, 2005. FAIGENBAUM, A.D.; KANG, J.; McFARLAND, J.; BLOOM, J.M.; MAGNATTA, J. Acute effects of different warmup protocols on anaerobic performance in teenage athletes. Pediatric Exercise Science, Champaign, v.18, p.64-75, 2006. 
FAIGENBAUM, A.D.; McFARLAND, J.; SCHWERDTMAN, J.A.; RATAMESS, N.A.; KANG, J.; HOFFMAN, J. Dynamic warm-up protocols, with and without a weighted vest, and fitness performance in high school female athletes. Journal Athletic Training, Dallas, v.41, p.357-63, 2006.

FAVERO, J.P.; MIDGLEY, A.W.; BENTLEY, D.J. Effects of an acute bout of static stretching on $40 \mathrm{~m}$ sprint performance: influence of baseline flexibility. Research in Sports Medicine, London, v.17, n.1, p.50-60, 2009.

FIELD, A. Discovering statistics using SPSS. 2nd. ed. London: Sage, 2005.

FLETCHER, I.M.; ANNESS, R. The acute effects of combined static and dynamic stretch protocols on fifty-meter sprint performance in track-and-field athletes. Journal of Strength and Conditioning Research, Champaign, v.21, n.3, p.784-7, 2007. FLETCHER, I.M.; JONES, B. The effect of different warm-up stretch protocols on 20 meter sprint performance in trained rugby union players. Strength and Conditioning Research, Champaign, v.18, n.4, p.885-8, 2004.

GELEN, E. Acute effects of different warm-up methods on sprint, slalom dribbling, and penalty kick performance in soccer players. Journal of Strength and Conditioning Research, Champaign, v.24, n.4, p.950-6, 2010.

GUISSARD, N.; DUCHATEAU, T.; HAINAUT, K. Mechanisms of decreased motoneurone excitation during passive muscle stretching. Experimental Brain Research, Berlin, v.137, p.163-9, 2001.

. Muscle stretching and motorneuron excitability. European Journal of Applied Physiology, Berlin, v.58, p.4752, 1988.

HEDGES, L.V.; OLKIN, I. Statistical methods for meta-analysis. New York: Academic Press, 1985.

HERMAN, S.L; SMITH, D.T. Four-week dynamic stretching warm-up Intervention elicits longer-term Performance benefits. Journal of Strength and Conditioning Research, Champaign, v.22, n.4, p.1286-97, 2008.

HOUK, J.C.; CRAG,O P.E.; RYMER, W.Z. Functional properties of the Golgi tendon organs. In: DESMEDT, J.E. (Ed.). Progress in clinical neurophysiology. Basel: Karger, 1980. p.33-43.

KISTLER, B.M.; WALSH, M.S.; HORN, T.S.; COX, R.H. The acute effects of static stretching on the sprint performance of collegiate men in the 60- and 100-m dash after a dynamic warm-up. Journal of Strength and Conditioning Research, Champaign, v.24, n.9, p.2280-4, 2010.

KOHN, A,F.; FLOETER, M.K.; HALLETT, M. Presynaptic inhibition compared with homosynaptic depression as an explanation for soleus H-reflex depression in humans. Experimental Brain Research, Berlin, v.116, p.375-80, 1997.

KOKKONEN, J; NELSON, A.G.; CORNWELL, A. Acute muscle stretching inhibits maximal strength performance. Research Quarterly for Exercise and Sport, Washington, v.69, p.411-5, 1998.

KRAEMER, W.J.; MAZZETTI, S.A.; NINDL, B.C.; GOTSHALK, L.A.; VOLEK, J.S.; BUSH, J.A.; ET AL. Effect of resistance training on women's strength/power and occupational performances. Medicine and Science in Sports and Exercise, Madison, v.33, p.1011-25, 2001

LITTLE, T.; WILLIAMS, A.G. Effects of differential stretching protocols during warm-ups on high-speed motor capacities in professional soccer players. Strength and Conditioning Research, Champaign, v.20, p.203-7, 2006.

LUNDBERG, A.; MALMGREN, K.; SCHOMBURG, E.D. Role of joint afferents in motor control exemplified by effects on reflex pathways from Ib afferents. Journal of Physiology, London, v.284, p.327-43, 1978.

McNAIR, P.J.; STANLEY, S.N. Effect of passive stretching and jogging on the series elastic muscle stiffness and range of motion of the ankle joint. British Journal of Sports Medicine, London, v.30, p.313-7, 1996.

MAGNUSSON, S.P.; RENSTROM, P. The European College of Sports Science position statement: the role of stretching in sports. European Journal of Sports Science, v.6, p.87-91, 2006.

MAHER, C.G.; SHERRINGTON, C.; HERBERT, R.D.; MOSELEY, A.M.; ELKINS M. Reliability of the PEDro scale for rating quality of randomized controlled trials. Physical Therapy, Alexandria, v.83, p.713-21, 2003

MIDDLETON LE, WENGER HA. Effects of menstrual phase on performance and recovery in intense intermittent activity. European Journal of Applied Physiology, Berlin, v.96, p.53-58, 2006.

MAIA, J.A.R.; GARGANTA, R.M.; SEABRA, A.; LOPES, V.P.; PRISTA, A.; FREITAS, D. Uma nota didáctica breve no uso esclarecido de procedimentos estatísticos em análise de dados repetidos no tempo: um estudo guiado para investigadores das ciências do desporto. Revista Portuguesa de Ciências do Desporto, Porto, v.4, n.3, p.115-33, 2004.

MARTIN, B.J.; ROBINSON, S.; WIEGMAN, D.L.; AULICK L.M. Effect of warm-up on metabolic responses to strenuous exercise. Medicine and Science in Sports, Madison, v.7, p.146-9, 1975.

McHUGH, M.P.; COSGRAVE, C.H. To stretch or no to stretch: the role of stretching in injury prevention and performance. Scandinavian Journal of Medicine \& Science in Sports, Copenhagen, v.20, p.169-81, 2010.

MURPHY, D.R. Dynamic range of motion training: An alternative to static stretching. Chiropractic Sports Medicine, v.8, p.59-66, 1994. 
MORSE, C.I.; DEGENS, H.; SEYNNES, O.R.; MAGANARIS, C.N.; JONES, D.A. The acute effect of stretching on the passive stiffness of the human gastrocnemius muscle tendon unit. The Journal of Physiology, Oxford, v.586, n.1, p.97-106, 2008. NEEDHAM, R.A.; MORSE, C.I.; DEGENS, H. The acute effect of different warm-up protocols on anaerobic performance in elite youth soccer players. Journal Strength and Conditioning Research, Champaign, v.23, n.9, p.2614-20, 2009. NELSON, A.G.; DRISCOLL, N.M.; LANDIN, D.K.; YOUNG, M.A.; SCHEXNAYDER, I.C. Acute effects of passive muscle stretching on sprint performance. Journal of Sports Science, London, v.23, p.449-54, 2005.

RHEA, M.R. Determining the magnitude of treatment effects in strength training research through the use of thr effect size. Journal of Strength and Conditioning Research, Champaign, v.18, n.4, p.918-20, 2004.

REILLY, T.; MORRIS, T.; WHYTE, G. The specificity of training prescription and physiological assessment: a review. Journal of Sports Science, London, v.27, n.6, p.575-89, 2009.

ROBINSON, K.L.; McCOMAS, A.J.; BELANGER, A.Y. Control of soleus motoneuron excitability during muscle stretch in man. Journal of Neurology, Neurosurgery, and Psychiatry, London, v.45, p.699-704, 1982.

ROSENBAUM, D.; HENNIG, EM. The influence of stretching and warm-up exercises on Achilles tendon reflex activity. Journal of Sport Science, London, v.13, p.481-90, 1995.

RUDOMIN, P. Presynaptic inhibition of muscle spindle and tendon organ afferents in mammalian spinal cord. Trends in Neurosciences, Barking, v.13, p.499-505, 1990.

SAFRAN, M.R.; SAEBER, A.V.; GARRETT JUNIOR, W.E. Warm up and muscular injury prevention: an update. Sports Medicine, Auckland, v.8, p.239-49, 1989.

SAYERS, A.L.; FARLEY, R.S.; FULLER, D.K.; JUBENVILLE, C.B.; CAPUTO, J.L. The effect of static stretching on phases of sprint performance in elite soccer players. Journal Strength and Conditioning Research, Champaign, v.22, n. 5, p.1416-21, 2008. SEKIR, U.; ARABACI, R.; AKOVA, B.; KADAGAN, S.M. Acute effects of static and dynamic stretching on leg flexor and extensor isokinetic strength in elite women athletes. Scandinavian Journal of Medicine \& Science in Sports, Copenhagen, v.20, n.2, p.268-81, 2010.

SHELLOCK, F.G.; PRENTICE, W.E. Warming-up and stretching for improved physical performance and prevention of sports-related injuries. Sports Medicine, Auckland, v.2, n.2, p.267-78, 1985.

SHORTEN, M.R. Muscle elasticity and human performance. Medicine and Science in Sports and Exercise, Madison, v.25, p.1-18, 1987. SIATRAS, T.; PAPADOPOULOS, G.; MAMELETZI, D.; GERODIMOS, V.; KELLIS, S. Static and dynamic acute stretching effect on gymnasts' speed in vaulting. Pediatric Exercise Science, Champaign, v.15, p.383-91, 2003.

SIM, A. Y.; DAWSON, B.T.; GUELFI, K. J.; WALLMAN, K. E.; YOUNG, W. B. Effects of static stretching in warm-up on repeated sprint performance. Journal Strength and Conditioning Research, Champaign, v.23, n.7, p.2155-62, 2009. STAPLETON, C.; SCOTT, M.A.; ATKINSON, G. The 'So What' Factor: statistical versus cinical significance. Internetional Journal of Sports Medicine, Stuttgart, v.30, p.773-4, 2009.

STEWART, A.S.; ADAMS, R.; ALONSO, A.; VAN KOESVELD, B.; CAMPBELL, C.S. Warm up or stretch as preparation for sprint performance? Journal of Science and Medicine in Sport, Victoria, v.10, n.6, p.403-10, 2007.

STONE, M.; RAMSEY, M. W.; KINSER, A. M.; O'BRYANT, H. S.; AYERS, C.; SANDS, W.A. Stretching: acute and chronic? The potential consequences. Strength and Conditioning Journal, Champaign, v.28, n.6, p.66-74, 2006.

TAYLOR, K, L.; SHEPPARD, J.M.; LEE, H.; PLUMMER, N. Negative effect of static stretching restored when combined with a sport specific warm-up component. Journal of Science and Medicine in Sport, Victoria, v.12, p.657-61, 2009. THOMAS, J.; FRENCH, K.. The use of meta-analysis in exercise and sport: a tutorial. Research Quarterly for Exercise and Sport, Washington, v.57, p.196-204, 1986.

WILSON, G.J.; MURPHY, A.J.; PRYOR, J.F. Musculotendinous stiffness: its relationship to eccentric, isometric, and concentric performance. Journal of Applied Physiology, Bethesda, v.76, p.2714-9, 1994.

WINCHESTER, J.B.; NELSON, A.G.; LANDIN, D.; YOUNG, M.A.; SCHEXNAYDER, I.C. Static stretching impairs sprint performance in collegiate track and field athletes. Journal Strength and Conditioning Research, Champaign, v.22, p.13-9, 2008.

WONG, P.L.; LAU, P.W.C.; MAO, D.W.; WU, Y.Y.; BEHM, D.G.; WISLOFF, U. Three days of static stretching within a warm-up does not affect repeated-sprint ability in youth soccer players. Journal of Strength and Conditioning Research, Champaign, v.25, n.3, p.838-45, 2011.

ZATSIORSKY, V.M.; KRAEMER, W.J. Prática e ciência do treinamento de força. São Paulo: Phorte, 2008. 


\section{Agradecimentos}

Os autores agradecem aos consultores que avaliaram este trabalho. Certamente, o mesmo ganhou clareza e qualidade após os seus pareceres.

Fabrício Boscolo Del Vecchio

ENDEREÇO

Grupo de Pesquisas em Treinamento Esportivo e Desempenho Físico

Escola Superior de Educação Física

Universidade Federal de Pelotas

R. Luiz de Camões, 625

96055-630 - Pelotas - RS - BRASIL

Recebido para publicação: 14/03/2011

Revisado: 21/07/2011

Aceito: 05/09/2011

e-mail:fabricio_boscolo@uol.com.br

Rev. bras. Educ. Fís. Esporte, São Paulo, v.25, n.4, p.567-81, out./ dez. 2011 • 581 